\title{
Development of the Evaluation Form for Expert Inspections of Web Portals
}

\author{
Andrina Granić ${ }^{1}$, Ivica Mitrović ${ }^{2}$, and Nikola Marangunić ${ }^{1}$ \\ ${ }^{1}$ Faculty of Science, University of Split, Nikole Tesle 12, 21000 Split, Croatia \\ \{andrina.granic, nikola.marangunic\} @pmfst.hr \\ ${ }^{2}$ Arts Academy, University of Split, Glagoljaška bb, 21000 Split, Croatia \\ ivica.mitrovic@umas.hr
}

\begin{abstract}
Web portals are a special breed of web sites, providing a large and diverse user population with a blend of information, services and facilities. Due to the lack of heuristics for the design and evaluation of general portals, we have conducted an experimental work to create research instrument for expert inspections. A set of 36 equally-levelled guidelines was developed to measure the hypothetical usability categories which reflect the four most distinctive elements of the contemporary web design process. The Portal Guidelines for expert inspections prove valuable to both novice inspectors and HCI experts, but further research will be required to validate the findings of this study.
\end{abstract}

Keywords: usability, expert inspection, Portal Guidelines, web portal.

\section{Introduction}

The idea of web portal is to collect information from different sources and create a single point of access to information, functions and services that are relevant to person's work or personal interests [1]. Due to specifics of portals as web sites, here primarily addressing their complex and hybrid structure and media specificities along with diversity of user population, their tasks and workflows, usability issues are crucial for their design. Yet many current web portals suffer from problems related to low usability since in many portal projects usability is an afterthought or is completely ignored in others. Usability assessment is often seen as too expensive, difficulty to accomplish, too specialized or something to address after testing all the "functionality" [2]. Consequently the efficient evaluation of web portals, and websites in general [3], has become a point of concern for both practitioners and researches.

Horizontal information (broad-reach and news) portals, also called general, are nowadays the most visited Croatian web sites [4]. Whether such portals do indeed reach their aim of facilitating users' access to diverse resources at the same time targeting the entire Internet community and, if so, to which extent, needs further investigation. To evaluate how easy to use horizontal information portals are, we have conducted a series of experiments that employed a range of usability assessment methods [5]. Unlike most studies related to web portal evaluations which usually rely on user-based surveys ( $c f$. [3] as well), our approach employed both empirical and 
analytical assessment. Such methodology is in line with general assumption that we should not rely on isolated evaluations, but instead make use of complementing usability techniques and of people with different expertise whom should be involved [6]. Our experience indicated that the chosen research instruments, measures and methods for web portal usability testing were adequate. Conversely, although showing considerable potential, analytical assessment raised some concerns. With the intention to employ expert inspections to complement rather than replace usability testing, the analytical evaluation was additionally checked up.

Due to the lack of guidelines/heuristics for the design and evaluation of general web portals, we have conducted an experimental work to develop a heuristic evaluation form for horizontal information portals. The work presented in this paper is based on our previous study when Nielsen's usability heuristics, as a set of ten key principles [7], was explained and adjusted to the general portal usage. As additional clarifications to each principle, a series of auxiliary guidelines concerning portal design were also provided, $c f$. e.g. [8]. Comprehensive quantitative and qualitative analysis of the acquired data revealed that some of the Nielsen's principles showed poor applicability in the web portal context. As a result, a set of seven general principles was prepared, each supplement with reduced and more helpful series of guidelines. Once again, obtained data was quantitative and qualitative analysed, taking into consideration relevant information acquired not only for the principles, but for all additional auxiliary guidelines. At this point our goal was to identify the most relevant heuristics/guidelines for web portal evaluation in order to create a heuristic evaluation form for the expert inspection of general portals.

\section{Heuristics for Expert Reviews}

Portal Guidelines, as a helpful research instrument for expert inspections was developed to measure the hypothetical usability categories which reflect the four most distinctive elements of the contemporary web design process. The categories also reflect actual design practice in general portals' development specifically their information architecture (INFO; 15 guidelines), navigation (NAV; 11 guidelines), layout/visual identity (VIS; 9 guidelines) and interactivity i.e. web specific practices (INT; 9 guidelines). Detailed quantitative and qualitative analysis of the guidelines was performed in our previous published experimental work. We have started with more than 100 guidelines and after comprehensive empirical evaluations the number of guidelines was reduced. As a result, a list of 44 guidelines was defined. Table 1 depicts examples of guideline statements organized in four categories. To explore reliability and validity of the evaluation form, the list was given to a group of 31 "instant/novice" [9] inspectors, chosen among computer science graduate and postgraduate students, and experienced practitioners from the field of web design.

General portal tportal (www.tportal.hr) was evaluated against the set of guidelines. A 5-point Likert-type scale asked the inspectors to rate the degree to which they agree with the guideline statements. Inspectors were asked to fill out an on-line version of the heuristic evaluation form. After two weeks of data collection, all 31 responses were gathered and analyzed. 
Table 1. Examples of guideline statements and their placement in four categories

\begin{tabular}{cl}
\hline Guideline category & \multicolumn{1}{c}{ Guideline statement } \\
\hline INFO & Thematic categories are clearly and simply organized \\
NAV & The navigation (horizontal or vertical) is positioned in consistent way \\
VIS & Commercials are avoided from the central part of the portal/screen \\
INT & Long and complicated registration is avoided \\
\hline
\end{tabular}

To estimate the internal consistency reliability of the scores, the Cronbach alpha coefficient was calculated for the initial set of 44 guidelines based on the sample of 31 novice inspectors. Classical item analysis was conducted on the items of the form to determine whether some items were negatively affecting the reliability of the scales. Elimination of eight "weakest" items resulted in reliability increase from 0.60 to 0.83 . Because the Cronbach's alpha values were above the conventional level of 0.7 [10], the set of guidelines is considered to exhibit adequate reliability.

The first step in the exploratory factor analysis was to estimate the number of factors in the heuristic evaluation form. The Kaiser-Gutman criterion (eigenvalue > 1) indicated that are more than four factors in the evaluation form. A principal factor analysis with varimax rotation (with Kaiser Normalization procedure) was conducted to ensure that guidelines for the same construct measure the particular category, while the guidelines for the other construct measure another one. Four factors that have explained $52.7 \%$ of variance in all items were extracted. The rotated factor matrix showed all the guidelines loaded on the particular latent constructs. Overall, the factors' structure in the factor analysis was not in agreement with the hypothetical structure of the developed Portal Guidelines.

\section{Discussion and Conclusion}

This paper briefly reports on the development of the Portal Guidelines as a valuable research instrument for the expert inspections of web portals. The designed heuristic evaluation form was created to measure the hypothetical usability categories which reflect actual work practice and characteristic components of the current web design process. At the beginning of this study of horizontal information web portals we assumed that the initial set of 44 guidelines could be grouped into four categories that reflect today's web design practice. After analyzing the internal guidelines' consistency reliability, we have obtained 36 guidelines that have shown reliability in the context of the research.

We have analyzed the potentially applicable situation with four categories of guidelines. Principal component analysis and comparison with the hypothetical categories has revealed that the guidelines are unevenly distributed in the four component categories. In fact, significant distinctiveness between categories could not be confirmed; specifically categories do not appear to be homogeneous - guidelines from these component categories could not be coupled in a reasonable/logical way. Therefore, we could assume that the factor analysis has not confirmed our hypothesis that the proposed set of guidelines could be categorized according to such criteria. On the contrary, the set of Portal Guidelines should be taken into account altogether. For 
that reason, the developed evaluation form is composed of 36 equally-levelled guidelines that should provide an insight into the overall usability of the portal. The additional analysis of individual guidelines which provided especially good or bad results could further interpret the specific elements of general web portal usability.

Finally we could conclude that the evaluation form for the expert inspection of general web portals has been successfully developed; the created Portal Guidelines are usable for both novice inspectors and HCI experts. A lot of efforts have gone into the direction of specializing general usability heuristics and validating the new proposed heuristic set. However, it would be also valuable to understand whether the defined evaluation form leads the inspectors to identify severe problems and if some categories of problems are privileged while others are neglected. The severity of the identified problems in particular is an important indicator about how good the heuristics are guiding the inspectors in the analysis of the applications, thus in problem discovering. Additionally, it would be interesting to compare the results of the inspection (the set of identified problems) with the results of an evaluation performed through a different set of heuristics or even a different assessment method.

Acknowledgments. This work has been carried out within project 177-0361994-1998 Usability and Adaptivity of Interfaces for Intelligent Authoring Shells funded by the Ministry of Science and Technology of the Republic of Croatia.

\section{References}

1. Beringer, J., Lessmann, C., Waloszek, G.: SAP AG - Generic Portal Pages - What Do Most Portals Need(May 21,2001),

http://www. sapdesignguild.org/editions/edition3/generic_page s.asp

2. Jensen, J.J., Skov, M.B., Stage, J.: The WPU Project: Web Portal Usability. ERCIM News 78 (2009)

3. Chiou, W.-C., Lin, C.-C., Perng, C.: A strategic framework for website evaluation based on a review of the literature from 1995-2006. Information \& Management 47, 282-290 (2010)

4. Digitalna Mreža (2011), http: / / www .digitalnamreza.com/

5. Granić, A., Mitrović, I., Marangunić, N.: Exploring the Usability of Web Portals: A Croatian Case Study. International Journal of Information Management (2010), doi:10.1016/j.ijinfomgt.2010.11.001

6. Sears, A., Jacko, J.: The Human-Computer Interaction Handbook: Fundamentals, Evolving Technologies and Emerging Applications. In: Human Factors and Ergonomics, 2nd edn. (2008)

7. Nielsen, J.: Heuristic Evaluation. In: Nielsen, J., Mack, R. (eds.) Usability Inspection Methods, pp. 25-64. John Wiley and Sons Inc., New York (1994)

8. Sharp, H., Rogers, Y., Preece, J.: Heuristics for Websites (2007), http://www.idbook.com/catherb/Website_heurs.php

9. Bolchini, D., Garzotto, F.: Quality and Potential for Adoption of Web Usability Evaluation Methods: An Empirical Study on MILE+. Journal of Web Engineering 7(4), 299-317 (2008)

10. Nunnally, J.C.: Psychometric theory. McGraw-Hill, New York (1978) 